\title{
Syk-Targeted, a New 3-Arylbenzofuran Derivative EAPP-2 Blocks Airway Inflammation of Asthma- COPD Overlap in vivo and in vitro
}

\author{
Shuyi Li \\ Yang Hui \\ Jiqiao Yuan \\ Ziqian Zhang \\ Xuyu Li \\ Nan Fang \\ Mingbao Lin (D) \\ Qi Hou (D)
}

Institute of Materia Medica, Chinese Academy of Medical Sciences \& Peking Union Medical College, Beijing, People's Republic of China
Correspondence: Mingbao Lin; Qi Hou Tel +86-10-63|65|92; +86-10-63165|9| Email mingbaolin@imm.cams.cn; houq@imm.ac.cn
Introduction: Asthma-chronic obstructive pulmonary (COPD) overlap (ACO) coexists with asthma and COPD syndrome characteristics, with more frequent exacerbations, heavier disease burden, higher medical utilization, and even lower quality of life. However, the ACO standard medications supported by evidence-based medicine have not yet appeared.

Methods: By using an ACO mouse model established previously and LPS-stimulated RAW264.7 macrophages in vitro, a potential therapeutic candidate, EAPP-2, was screened from derivatives of 3-arylbenzofuran, and its effect and mechanism on ACO inflammation were evaluated.

Results: EAPP-2 significantly alleviated airway inflammation in ACO mice and also inhibited the inflammatory reactions in LPS-induced RAW264.7 macrophages in vitro. Furthermore, EAPP-2 significantly inhibited the expression and phosphorylation of spleen tyrosine kinase (Syk), a common target regulating both eosinophils and neutrophils inflammation. In addition to this, EAPP-2 significantly down-regulates the expression of NF- $\mathrm{KB}$, $\mathrm{p}-\mathrm{NF}-\kappa \mathrm{B}$, and NLRP3 in vivo and in vitro. Moreover, by using specific inhibitors in vitro, it was validated that EAPP-2 targeted on Syk and then regulated its downstream NF- $\mathrm{B}$ and NLRP3.

Conclusion: EAPP-2 is shown to be a potentially useful therapeutic candidate for ACO, and its mechanism is at least partially achieved by targeting on Syk and then inhibiting NF- $\mathrm{B}$ or NLRP3. Moreover, this study suggests that Syk may be a potentially effective target for ACO therapy.

Keywords: EAPP-2, asthma-COPD overlap, ACO, Syk, NF- $\kappa B$, NLRP3

\section{Introduction}

Asthma and chronic obstructive pulmonary disease (COPD), two clinical frequently - occurring chronic airway inflammatory diseases, are caused by different inflammatory pathological mechanisms. However, with many similarities in clinical symptoms, sometimes it is hard to diagnose asthma or COPD clearly. Furthermore, a considerable number of patients coexist with the problems of asthma and COPD. So that asthma-COPD overlap (ACO), referring to patients "characterized by persistent airflow limitation with several features usually associated with asthma and several features usually associated with COPD," was used as a "description for clinical use" by the Global Initiative of Asthma (GINA) and the Global Initiative for Chronic Obstructive Pulmonary Disease (GOLD) in 2017. ${ }^{1,2}$ In clinical trials, ACO patients have more frequent exacerbations, heavier disease burden, higher 
medical utilization, and lower quality of life compared to those with asthma and COPD alone. ${ }^{3-6}$ However, besides the difficulty to diagnose, there is still a lack of exact specific and effective treatment of ACO. At present, the treatment of suspected ACO is mainly based on the treatment of severe asthma and COPD phenotype guidance, treated with inhaled glucocorticoids (ICS), long-acting $\beta 2$-agonist (LABA) and long-acting muscarinic antagonists (LAMA). Therefore, looking for specific drugs or methods for ACO treating has become particularly important and urgent.

A potential common target of asthma and COPD inflammation may also be an effective target for ACO therapeutic intervention. Syk, a $72 \mathrm{kD}$ non-receptor protein tyrosine kinase, widely present in various hematopoietic cells and some non-hematopoietic cells. ${ }^{7-9}$ Syk has been proved to be a potential therapeutic target for both asthma and COPD inflammation. ${ }^{10,11}$ In asthma, Syk is rapidly recruited and activated by FceRI-mediated signaling pathways in human basophils, leading to cell degranulation and histamine release. ${ }^{12}$ And in patients with COPD, Syk is one of the kinases required for neutrophils to form neutrophil extracellular traps (NET). ${ }^{13,14}$ Furthermore, previously, by using comparative RNA-Seq transcriptome analysis on pulmonary inflammation in an ACO mouse model, we also found that Syk was a potential mainly affected signaling pathways of inflammation in ACO mouse. ${ }^{15}$ Phosphorylated Syk activated via its $\mathrm{SH} 2$ domains binds to the dual phosphorylated immunoreceptor tyrosine-based activation motif (ITAM) ${ }^{16}$ and its phosphorylated tyrosine residues can be used as anchor points for ligands binding, then to trigger its downstream signal cascades, including NF- $\mathrm{KB}$ and NLRP $3,{ }^{17,18}$ which ultimately trigger an inflammatory response. Therefore, targeting on Syk seems to be an effective solution for ACO airway inflammation treatment.

Due to its diverse biological properties, benzofuran is the leading structure of many clinically approved drugs that are synthetic or naturally occurring substituted benzofuran derivatives. Some of the synthesized 2,2-bisaminomethylated aurone analogues exerted excellent antiinflammatory activity against TNF- $\alpha$ and IL- $6 .{ }^{19}$ Benzofuran derivatives also showed good antiinflammatory activity in rat carrageenan-induced paw edema and strong inhibitory activity of cyclooxygenase-2 (COX-2) enzyme. ${ }^{20-22}$ Based on these, we synthesized a 3-arylbenzofuran derivative EAPP-2 (Figure 1A), which exhibited better anti-inflammatory activity and inhibitory activity of Syk.

Therefore, in this study, the effects of EAPP-2 on ACO airway inflammation were evaluated in vivo and in vitro, and then its underlying mechanisms targeted on Syk/NF$\kappa \mathrm{B} / \mathrm{NLRP} 3$ pathway were investigated.

\section{Materials and Methods \\ Animals}

Male Balb/c mice weighting 20-22g were purchased from the Vital River Laboratory Animal Technology Co., Ltd (Beijing, China), housed in a specific pathogen-free room with a controlled environment $\left(23 \pm 2{ }^{\circ} \mathrm{C}, 40 \pm 10 \%\right.$ humidity, a 12-h light/dark cycle) and fed with standard chow and filtered tap water ad libitum. A All animals' experiments were approved by the Animal Care and Ethics Committee of the Institute of Materia Medica (IMM), Chinese Academy of Medical Sciences and Peking Union Medical College (CAMS and PUMC), approved by The Animal Care and Welfare Committee of IMM, CAMS and PUMC, and conformed to internationally accepted ethical standards.

\section{LPS + OVA + CS-Induced ACO in Mice}

Mice were randomly divided into six groups: Control, Model, EAPP-2 $(7.5,15$, and $30 \mathrm{mg} / \mathrm{kg}$, prepared by Prof Chunsuo Yao, the Institute of Materia Medica, Chinese Academy of Medical Sciences, Beijing, China), dexamethasone sodium phosphate injection group $(0.5 \mathrm{mg} / \mathrm{kg}$, DEX, Sinopharm Group Rongsheng Pharmaceutical Co., Ltd., Jiaozuo, China). On days 1, 7, and 14, mice were sensitized with an intraperitoneal injection of $30 \mu \mathrm{g}$ ovalbumin (OVA, Sigma-Aldrich, St. Louis, MO, USA) in 100 $\mu 1$ aluminum hydroxide gel (Meihua Chemical Co. Ltd., Shanghai, China). Then, the mice were intratracheally instilled with $30 \mu \mathrm{g}$ lipopolysaccharide (LPS, SigmaAldrich, St. Louis, MO, USA) in $50 \mu \mathrm{L}$ saline under light anesthesia on day 15 , and challenged by intratracheal instillation of $60 \mu \mathrm{g}$ OVA in $50 \mu \mathrm{L}$ saline on days 26 and 27, $30 \mu \mathrm{g}$ OVA plus $15 \mu \mathrm{g}$ LPS in $50 \mu \mathrm{L}$ saline on day 28 . Mice in the control group received the same volume of normal saline. On days $16-25$ and days $29-42$, mice were exposed to cigarette smoke for 60 min once a day in a small animal smoke inhalation microenvironment preparation system (MBL-1, the Institute of Materia Medica, Chinese Academy of Medical Sciences, Beijing, China) with the condition of smoke density at $300 \pm 50 \mathrm{ppm}$, and 


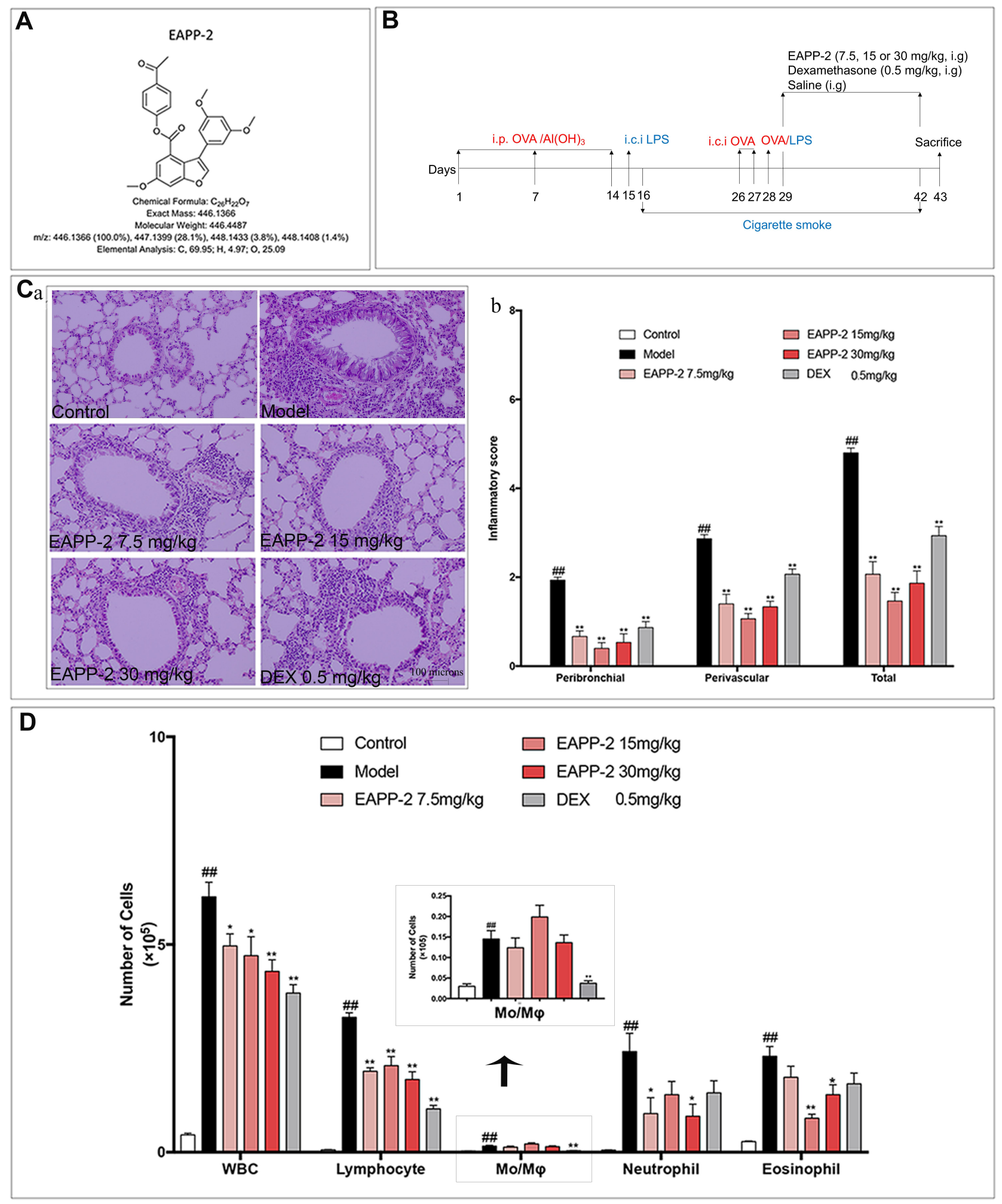

Figure I Effects of EAPP-2 on lung inflammatory cells infiltration in ACO mice. (A) Molecular structure of EAPP-2. (B) Timeline of the experimental protocol. (C) a: Representative images of H\&E staining of lung tissue (magnified $\times 100$ ); b: peribronchial and perivascular inflammatory infiltration scoring by a four-point scale (five independent slices from each mouse, $n=3$ ). (D) The inflammatory cell in $B A L F$ counts $(n=8)$. Data were shown as mean $\pm S E M ;{ }^{\#} P<0.0$ l compared with the control group; $* \mathrm{P}<0.05$ and $* * \mathrm{P}<0.01$ compared with the model group. 
oxygen concentration higher than $18.5 \%$. From day 29 , mice were administered by gavaging test compounds $1 \mathrm{~h}$ before CS exposure, once a day for 14 consecutive days (Figure 1B).

\section{Cell Counting in Bronchoalveolar Lavage Fluid (BALF)}

Twenty-four hours after the last treatment, mice were anesthetized with sodium pentobarbital $(0.8 \%)$ and sacrificed. The BALF was collected by cannulating the trachea and washing the lung with $800 \mu \mathrm{L}$ ice-cold phosphatebuffered saline (PBS) triply, centrifuged at $1000 \times g$ for $10 \mathrm{~min}$ at $4^{\circ} \mathrm{C}$, and then the supernatants were collected and stored at $-80^{\circ} \mathrm{C}$ for the determination of inflammatory factors. The pelleted cells were resuspended in $100 \mu \mathrm{L}$ PBS containing EDTA.2K $(5 \mathrm{mg} / \mathrm{mL})$ and determined using a hemocytometer (Mindray BC-5000 vet, Shenzhen, China).

\section{Lung Histomorphometry}

Lung tissues were collected and fixed in $10 \%$ formalin solution for 24 hours before embedding in paraffin. Four$\mu \mathrm{m}$ thick sections were stained with hematoxylin and eosin (H\&E) and observed under a light microscope (100×). Experienced blinded pathologists performed the histopathological studies, according to a four-point scoring scale. ${ }^{11}$

\section{Cell Culture and Treatment}

Macrophage cell line RAW264.7 was obtained from American Type Culture Collection (ATCC, Rockville, MD, USA) and cultured in Dulbecco's modified Eagle's medium (DMEM, Gibco, Carlsbad, CA, USA) supplemented with $10 \%$ heat-inactivated fetal bovine serum (FBS, SeraPro, Naila, Germany), $100 \mathrm{U} / \mathrm{mL}$ penicillin and 100 $\mu \mathrm{g} / \mathrm{mL}$ streptomycin in a $37{ }^{\circ} \mathrm{C}$ humidified incubator containing $5 \% \mathrm{CO}_{2}$.

The cells were plated into 96-well plates or culture dishes ( $60 \mathrm{~mm}$ in diameter) at a density of $4 \times 10^{5}$ cells $/ \mathrm{mL}$, and pre-incubated with EAPP-2 $(2.5,5$, and $10 \mu \mathrm{M}$, respectively), DEX (1 $\mu \mathrm{M})$, BAY61-3606 (an inhibitor of Syk, 0.5 $\mu \mathrm{M}$, Sigma, St Louis, MO, USA), BAY11-7082 (an inhibitor of NF-kB, $5 \mu$ M, Sigma, St. Louis, MO, USA), MCC950 (an inhibitor of NLRP3, $0.5 \mu \mathrm{M}$, Topscience Biotech Co., Ltd., Shanghai, China) for 1 hour, followed by stimulated with LPS ( $1 \mu \mathrm{g} / \mathrm{mL}$, Sigma, St. Louis, MO, USA) for $24 \mathrm{~h}$. The supernatants in 96-well plates were collected for cytokine analysis. And cells in culture dishes were used for Western blotting analysis.

\section{Nitrate Quantification by Griess Test}

The cells of RAW264.7 macrophages were seeded into 96well plates at a density of $2 \times 10^{5}$ cells $/$ well and pre-treated with EAPP-2 $(2.5,5$, and $10 \mu \mathrm{M}$, respectively) or DEX $(1 \mu \mathrm{M})$ for 1 $\mathrm{h}$, then stimulated with LPS $(1 \mu \mathrm{g} / \mathrm{mL})$ for $24 \mathrm{~h}$. Culture supernatants were collected and mixed with $100 \mu \mathrm{L}$ Griess reagent ( $1 \%$ sulfanilamide, $0.1 \% \mathrm{~N}-1$-naphthylenediamine dihydrochloride, and $2.5 \%$ phosphoric acid). The absorbance was measured at $570 \mathrm{~nm}$ with a microplate reader (Power Wave XS2, BioTek, Virginia, USA).

\section{Enzyme-Linked Immunosorbent Assay (ELISA)}

The cytokine levels in BALF and cell culture supernatants were measured by using mouse IL-4, IL-6, IL-1 $\beta$, IL-17A, IFN- $\gamma$, and TNF- $\alpha$ ELISA kits (BioLegend, San Diego, CA, USA). The cytokine levels in the serum were determined by total IgE ELISA kits (BioLegend) and IgG1 ELISA kits (Bethyl Laboratories, Montgomery, TX, USA). All of these according to the manufacturer's instructions, respectively.

\section{Western Blotting Analysis}

The lung tissues or cells cultured in vitro were lysed and centrifugated at $12,000 \times \mathrm{g}$ for $10 \mathrm{~min}$ at $4{ }^{\circ} \mathrm{C}$, and then the supernatants were removed and quantified via the BCA method (Solarbio, Beijing, China). Equal amounts of protein were separated by $10 \%$ sodium dodecyl sulfate-polyacrylamide gel electrophoresis (SDS-PAGE), then transferred onto polyvinylidene difluoride membranes (PVDF, Millipore, Bedford, MA, USA) and blocked with $5 \%$ skim milk for $1 \mathrm{~h}$ at room temperature. The membranes were first probed with specific primary antibodies against Syk (\#13198S), phospho-Syk (Y525/526)

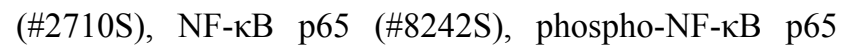
(Ser536) (\#3033S), NLRP3 (\#15101S), iNOS (\#13120S) and $\beta$-actin (\#4970) (Cell Signaling Technology, Boston, MA, USA) separately overnight at $4{ }^{\circ} \mathrm{C}$. After washing, they were incubated with horseradish peroxidaseconjugated anti-rabbit secondary antibodies for $1 \mathrm{~h}$ at room temperature and then visualized with an enhanced chemiluminescence kit (ECL, Thermo Scientific, Rockford, IL, USA) on a Chemical imaging system (Clinx Science Instruments Co., Ltd, Shanghai, China). 
The relative intensities of bands were quantified using Image-J software (National Institutes of Health, Bethesda, Maryland, USA).

\section{Statistical Analysis}

Data were expressed as mean \pm standard error of means (SEM), and statistically analyzed by using the Student's $t$-test for two groups comparison or one-way ANOVA for multiple groups, being conducted with Prism software version 7 (GraphPad Software, San Diego, CA, USA). A $\mathrm{P}$ value $<0.05$ was considered to be statistically significant.

\section{Results}

\section{EAPP-2 Attenuated Lung Inflammatory Cells Infiltration in ACO Mice}

By using H\&E staining, the inflammatory histological changes in the lungs were detected, and quantitatively scored. The results showed that a significant increase of inflammatory cells infiltrated into perivascular and peribronchial regions in ACO model mice compared with the mice in control group, while which were marked attenuated with EAPP-2 treatment $(\mathrm{P}<0.01)$, as well as with the DEX treatment (Figure 1C).

Accordingly, in BALF, compared with the mice in the control group, cell counts of the total leukocytes, lymphocytes, monocytes, neutrophils, and eosinophils were all significantly increased $(p<0.01$, Figure 1D). Consistent with the results of histological analysis, EAPP-2 treatment markedly decreased the inflammatory cells infiltration in BALF, including the total leukocytes, lymphocytes, neutrophils, and eosinophils, in which the inhibition of total leukocytes shown a dose-dependence $(p<0.05$ or 0.01$)$. Significant reductions were shown in the total leukocytes, lymphocytes, and monocytes with DEX treatment $(p<0.01)$.

\section{EAPP-2 Decreased the Levels of Inflammatory Cytokines in BALF and Serum Immunoglobulins of ACO Mice}

The levels of inflammatory cytokines in BALF were determined using ELISA, as well as serum total IgE and IgG1. In $\mathrm{ACO}$ mice, significantly increases of TNF- $\alpha$ (Figure 2A), IL-6 (Figure 2B), IL-17A (Figure 2C), IL-1 $\beta$ (Figure 2D), IL-4 (Figure 2E), IFN- $\gamma$ (Figure 2F) in BALF were detected $(\mathrm{P}<0.01)$. While they were significantly decreased with EAPP-2 treatment $(\mathrm{P}<0.05$ or 0.01 ), showing a dose-dependence in the inhibition of
IL-6, IL-17A, TNF- $\alpha$. Also, EAPP-2 $(15 \mathrm{mg} / \mathrm{kg})$ could significantly reduce the ratio of IL-4/IFN- $\gamma(\mathrm{P}<0.01$, Figure $2 \mathrm{G}$ ), indicating that EAPP-2 has a certain regulatory effect on Th2-type immune response. Among these, no significance was observed in the levels of IL-17A, TNF- $\alpha$, IL-1 $\beta$ with DEX treatment $(\mathrm{P}>0.05)$.

As also shown in immunoglobulins in the serum, EAPP-2 treatment significantly decreased the levels of total IgE (Figure 2H) and IgG1 (Figure 2I) in mice ACO, as well as DEX treatment $(\mathrm{P}<0.05$ or 0.01$)$.

\section{EAPP-2 Inhibited the Production of Inflammatory Cytokines in vitro}

The anti-inflammatory activity of EAPP-2 in vitro was investigated by using LPS-stimulated RAW264.7 macrophages. No significant cytotoxicity of EAPP-2 on RAW264.7 macrophages was observed from 2.5 up to $120 \mu \mathrm{M}$ concentrations (Data not shown). As shown in Figure 3, RAW264.7 macrophages stimulated with LPS markedly increased the production of NO (Figure 3A), IL-6 (Figure 3B), TNF- $\alpha$ (Figure $3 \mathrm{C}$ ), and IL-1 $\beta$ (Figure 3D), while that EAPP-2 pre-treated were significantly inhibited $(\mathrm{P}<0.01)$, and shown a dosedependent manner. These results suggested a significant antiinflammatory activity of EAPP-2 in vitro.

\section{EAPP-2 Inhibited the Expression and Activation of Syk and Its Downstream Signals in Lung Tissues of ACO Mice} In lung tissues of ACO mice, the protein expression of Syk (Figure 4A), p-Syk (Figure 4B), NF- $\kappa \mathrm{B}$ (Figure 4C), p-NF- $\kappa B$ (Figure 4D), NLRP3 (Figure 4E), and iNOS (Figure 4F) were significantly increased $(p<0.01)$, suggesting the activation of Syk and its downstream signals in ACO mice. And with EAPP-2 treatment, the expression

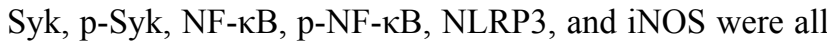
significantly inhibited, as well as DEX treatment $(p<0.05$ or 0.01). Collectively, the results suggested that a potential mechanism of EAPP-2 on ACO inflammations was via down-regulating the Syk pathway.

\section{EAPP-2 Inhibited the Expression and Activation of Syk and Its Downstream Signals in LPS- Stimulated RAW264.7 Macrophages}

Consistent with the in vivo results, EAPP-2 significantly inhibited the expression of Syk (Figure 5A), p-Syk (Figure 

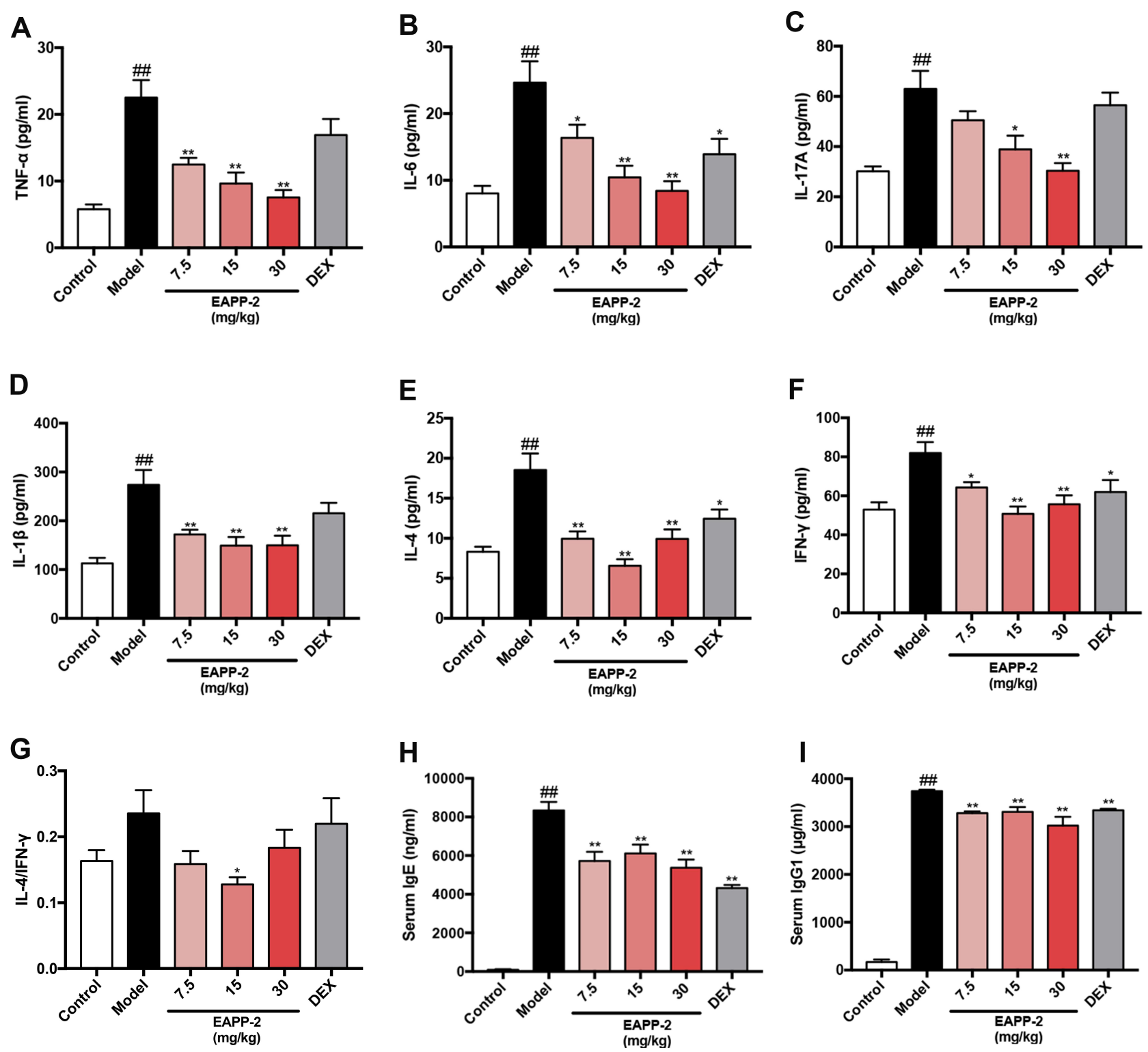

Figure 2 Effects of EAPP-2 on the levels of inflammatory cytokines TNF- $\alpha(\mathbf{A})$, IL-6 (B), IL-I7A (C), IL-I $\beta$ (D), IL-4 (E), IFN- $\gamma$ (F) and IL-4/IFN- $\gamma(\mathbf{G})$ in BALF, and serum immunoglobulins IgE $(\mathbf{H})$ and $\operatorname{lgGI}(\mathbf{I})$ of ACO mice $(\mathrm{n}=8)$. Data were shown as mean $\pm \mathrm{SEM}$; ${ }^{\#} \mathrm{P}<0.0 \mathrm{I}$ compared with the control group; $* \mathrm{P}<0.05$ and $* * \mathrm{P}<0.0 \mathrm{I}$ compared with the model group.

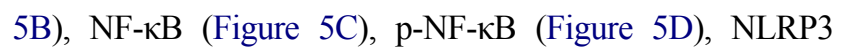
(Figure 5E) and iNOS (Figure 5F) in LPS-stimulated RAW264.7 macrophages with dose-dependent manners $(\mathrm{p}<$ 0.05 or 0.01$)$. While with DEX treatment, only a significant decrease was shown in NF-אB, NLRP3, and iNOS expression.

\section{Validation of That EAPP-2 Attenuating} ACO Inflammation via Potential

\section{Syk-Targeted in vitro}

Inhibitors of Syk (BAY61-3606), NF-kB (BAY11-7082), and NLRP3 (MCC950) were used to validated the potential Syk-targeted mechanism in vitro. As shown in Figure $6 \mathrm{~A}$ and $\mathrm{B}$, significant synergistic inhibited effects of EAPP-2 co-treatment with inhibitors of BAY61-3606, BAY11-7082, MCC950 have indicated on the production of IL-6 and TNF- $\alpha$ in LPS-induced RAW264.7 macrophages. Furthermore, Syk inhibitor BAY61-3606 significantly enhanced the inhibitory effects of EAPP-2 on Syk (Figure 6C) and p-Syk (Figure 6D) expression, while no enhanced inhibitory effects being shown in NF- $\mathrm{KB}$ inhibitor AY11-7082 and NLRP3 inhibitor MCC950 cotreatment. Syk inhibitor BAY61-3606 significantly inhib-

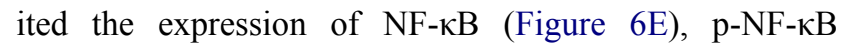



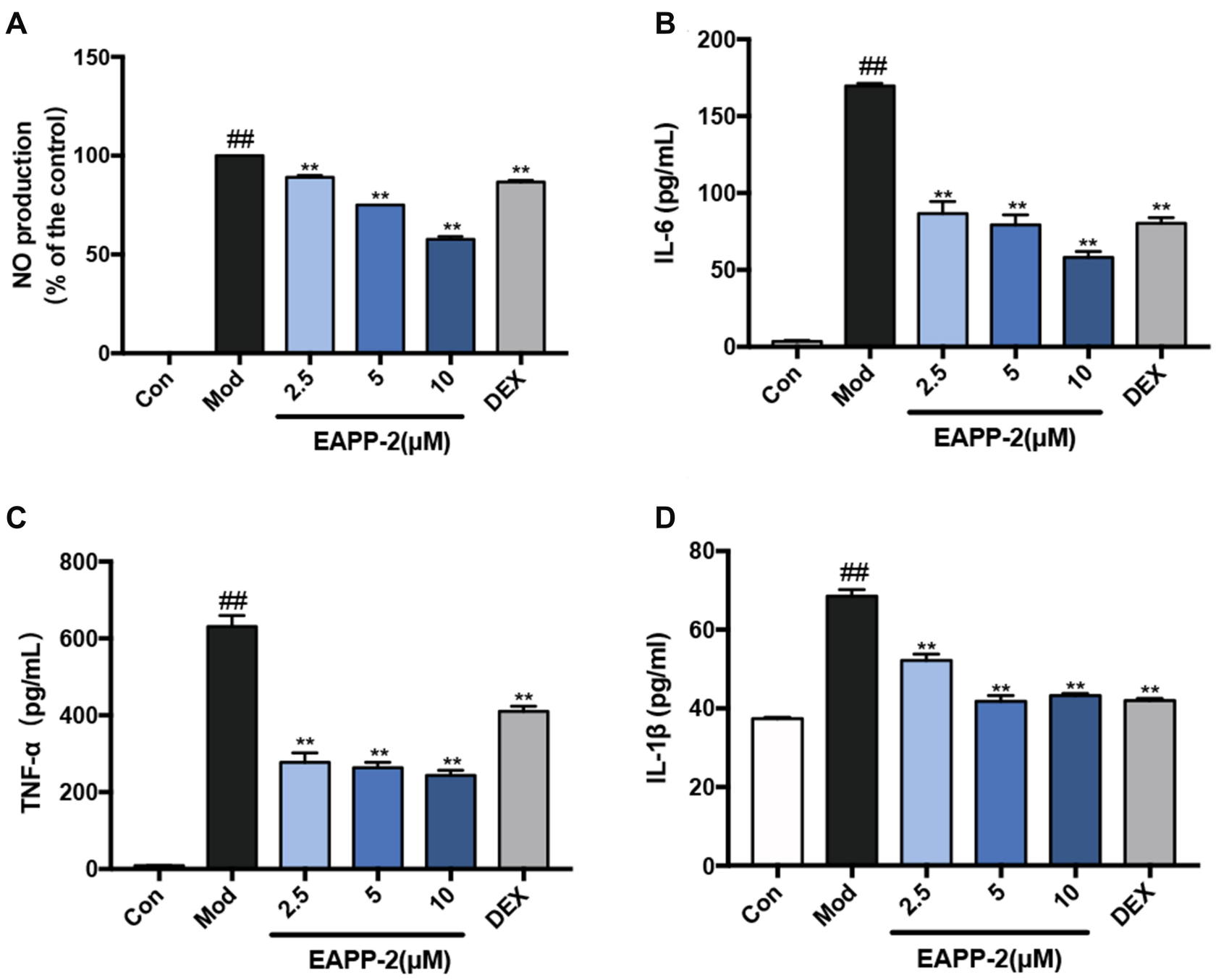

Figure 3 Anti-inflammatory activity of EAPP-2 on LPS-stimulated RAW264.7 macrophages in vitro. The levels of NO detected by Griess test (A), and the levels of IL-6 (B), TNF- $\alpha(\mathbf{C})$, and IL-I $\beta$ (D) in supernatants detected by ELISA ( $n=3$ independent experiments). Data were shown as mean \pm SEM; ${ }^{\#} \mathrm{P}<0.0 \mathrm{I}$ compared with the control group; **P $<0.01$ compared with the model group.

(Figure 6F), and NLRP3 (Figure 6G), and EAPP-2 cotreatment significantly enhanced the inhibitory effects. However, NLRP3 inhibitor MCC950 had no significant inhibitory effect on the expression and phosphorylation of syk and NF- $\kappa \mathrm{B}$. Collectively, these results indicated that EAPP-2 might potentially be targeted on Syk, and then regulating its downstream NF- $\kappa \mathrm{B} / \mathrm{NLRP} 3$ to attenuate ACO inflammation.

\section{Discussion}

ACO accounts for a certain proportion of asthma and COPD patients, with more frequent exacerbations, heavier disease burden, higher medical utilization, and even lower quality of life. However, for its clinical symptoms coexist with the problems of asthma and COPD, the ACO standard medications supported by evidence-based medicine have not yet appeared. At present, clinicians are forced to use trial and error to find the best treatment for ACO patients, inhaled glucocorticoids (ICS), long-acting 32 -agonist (LABA) and long-acting muscarinic antagonists (LAMA) were used mainly based on the treatment of severe asthma and COPD phenotype guidance. Therefore, specific drugs or methods for ACO treating become particularly urgent. In this study, by using the ACO mouse model we established previously, a potential therapeutic candidate, EAPP-2, was screened from derivatives of 3-arylbenzofuran. The results showed that EAPP-2 significantly alleviated airway inflammation in ACO mice and also inhibited the inflammatory reactions in LPSinduced RAW264.7 macrophages in vitro.

In ACO patients, coincided in the established ACO mice, there is a large increase in both sputum eosinophils 

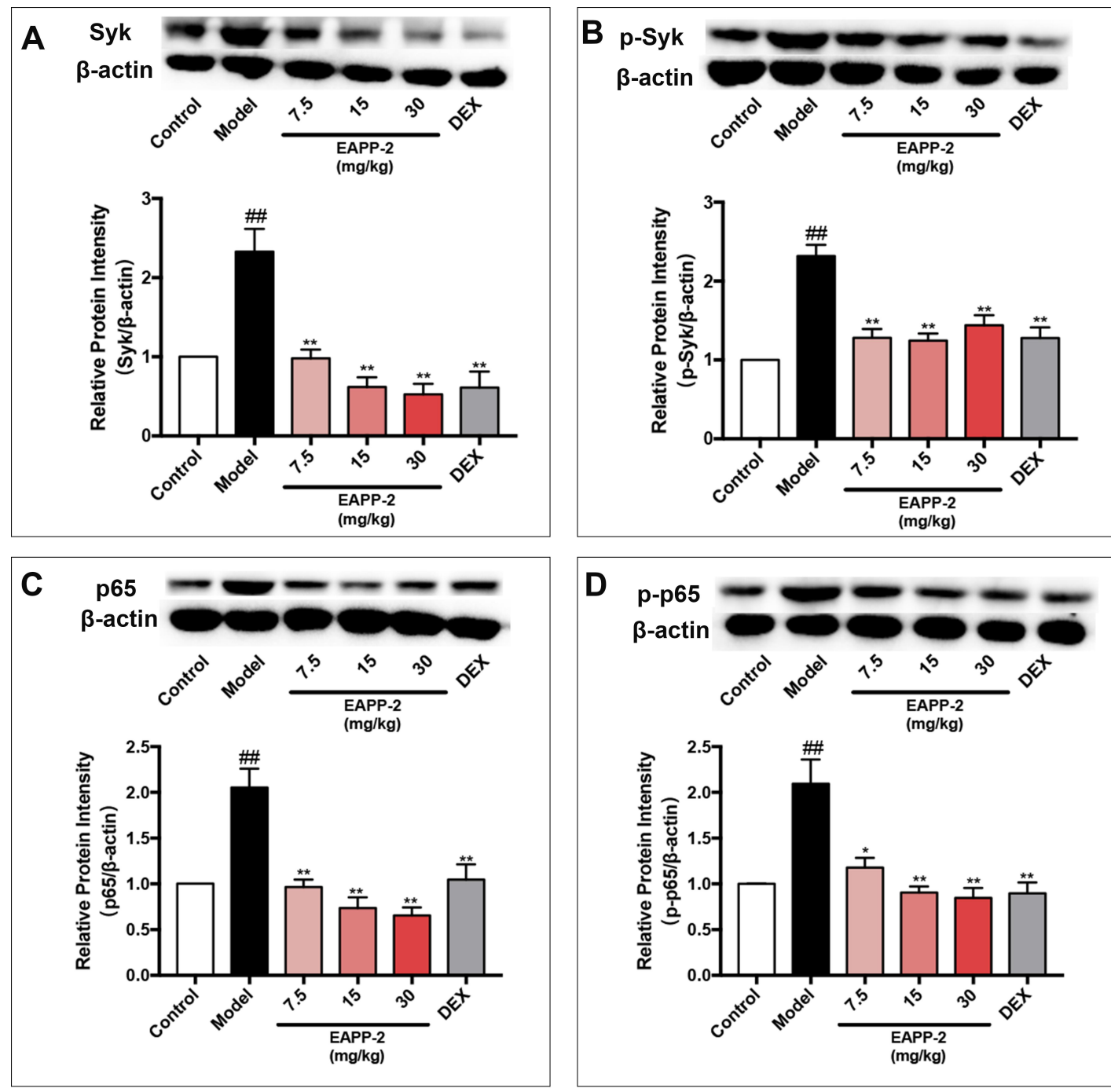

$\mathbf{E}$
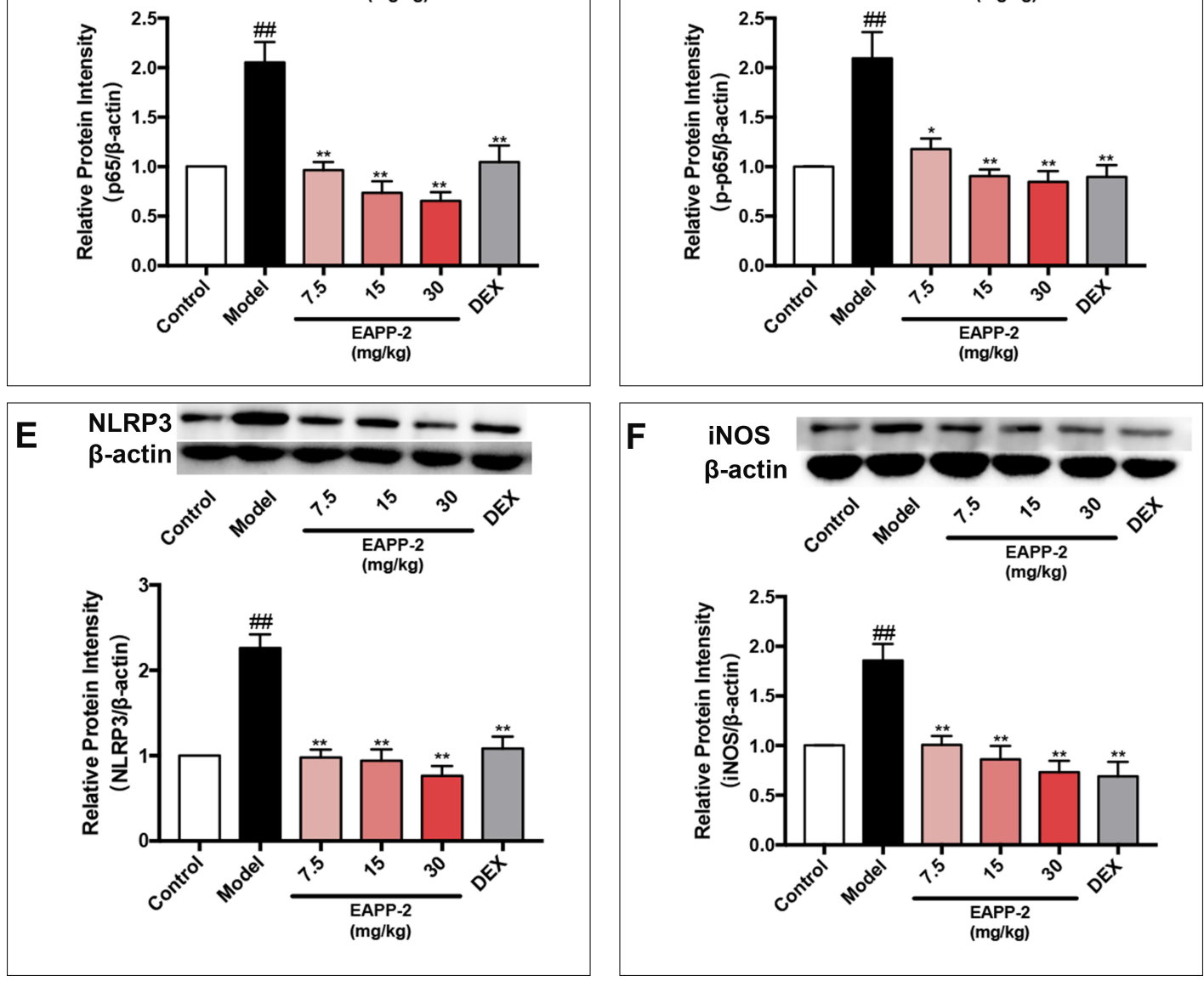

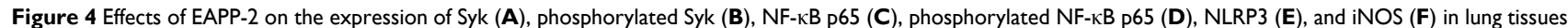
of ACO mice $(n=5)$. Data were shown as mean \pm SEM; ${ }^{\#} P<0.01$ compared with the control group; $* P<0.05$ and $* * P<0.01$ compared with the model group. 

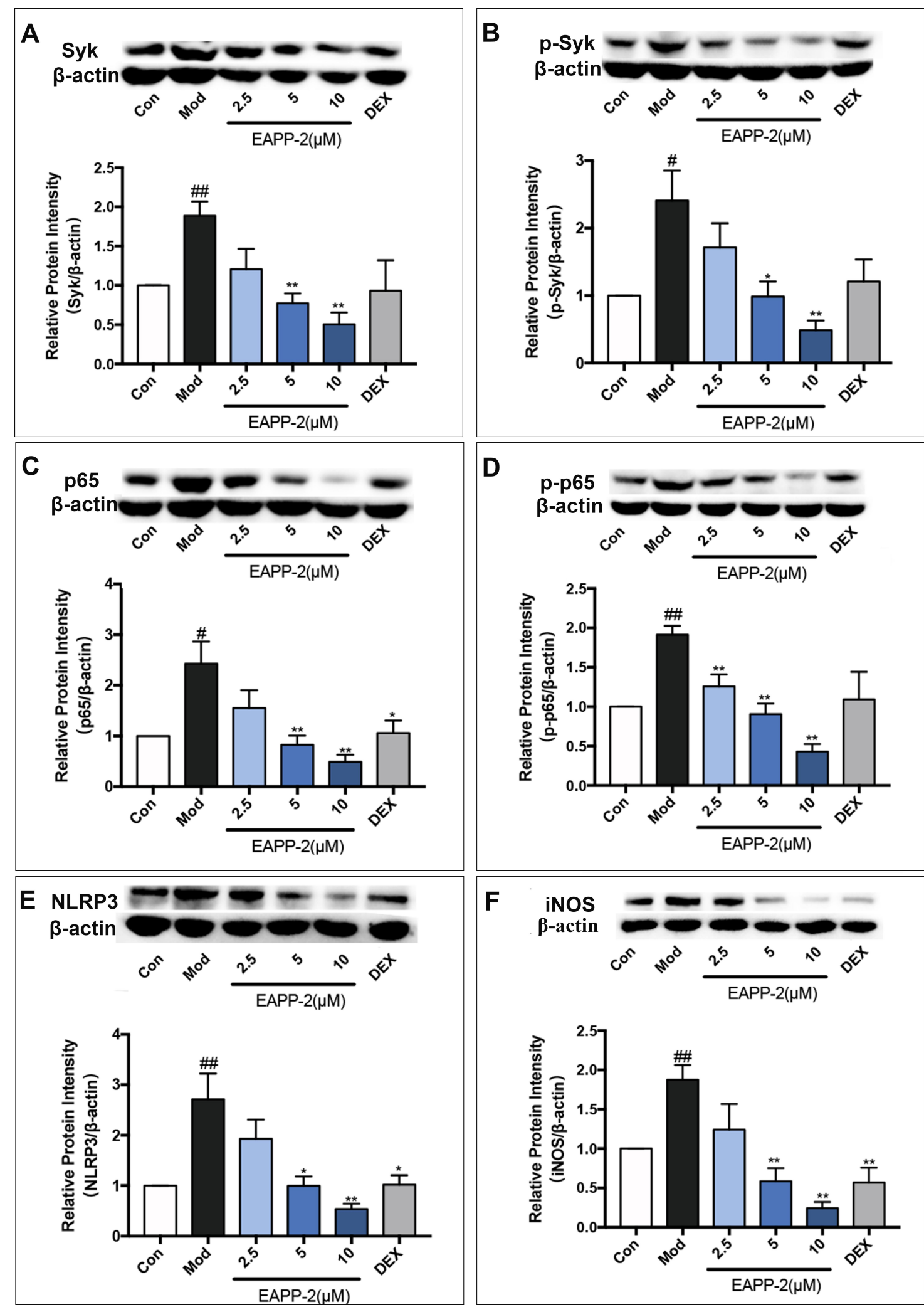

Figure 5 Effects of EAPP-2 on the expression of Syk (A), phosphorylated Syk (B), NF-kB p65 (C), phosphorylated NF-kB p65 (D), NLRP3 (E), and iNOS (F) in LPSstimulated RAW264.7 macrophages $(n=5)$. Data were shown as mean \pm SEM; ${ }^{\#} P<0.05$ and ${ }^{\# \#} P<0.01$ compared with the control group; ${ }^{*} P<0.05$ and $* * P<0.01$ compared with the model group. 

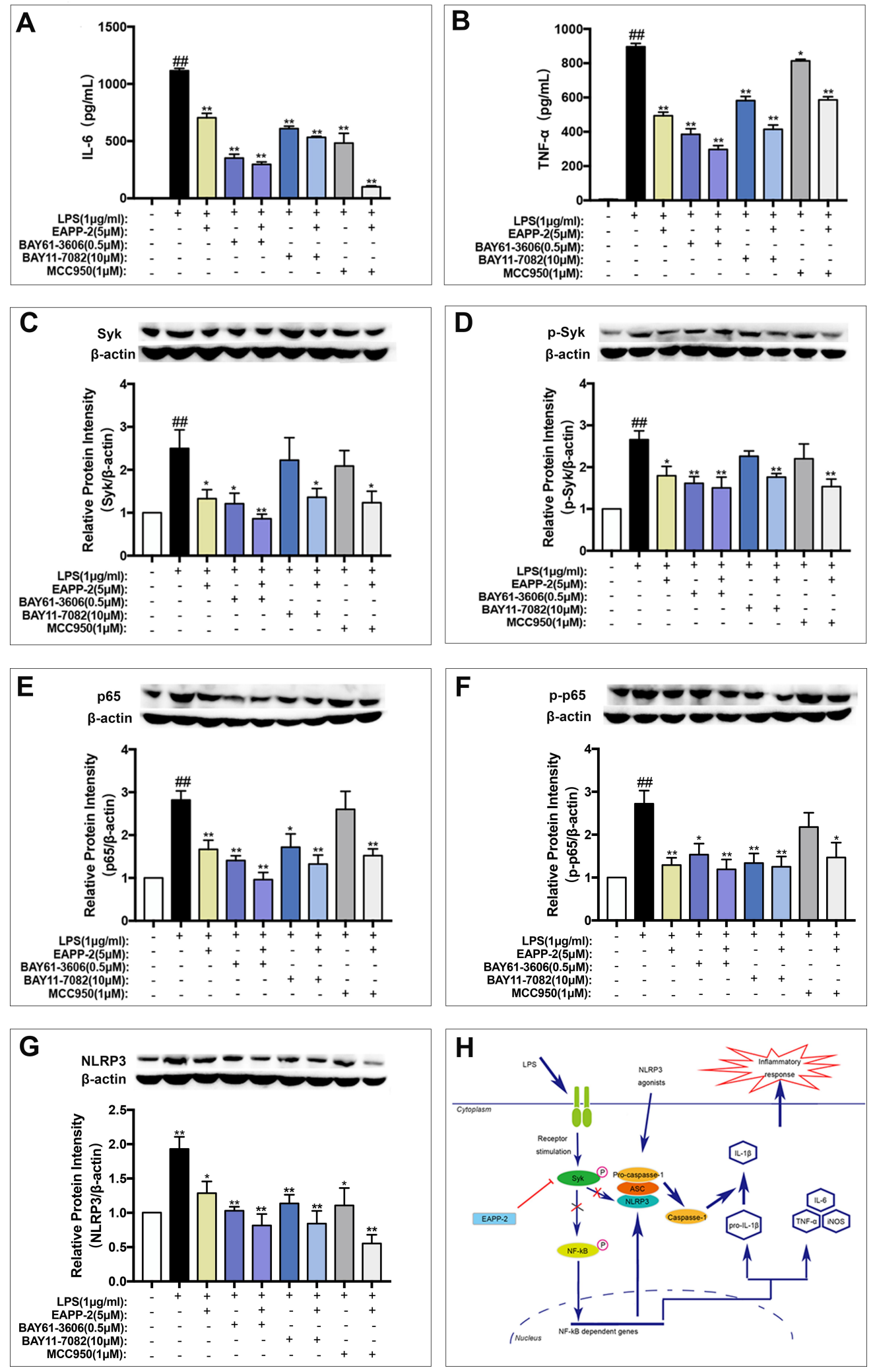

Figure 6 Validation of that EAPP-2 attenuating ACO inflammation via potential Syk-targeted in vitro. The production of IL-6 (A) and TNF- $\alpha$ (B) in supernatants of LPSstimulated RAW264.7 macrophages detected by ELISA ( $n=3$ independent experiments), and expressions of Syk (C), phosphorylated Syk (D), NF- $k B$ p65 (E), phosphorylated NF-kB p65 (F), and NLRP3 (G) $(n=5)$ in LPS- stimulated RAW264.7 macrophages detected by Western blotting. (H) Schematic diagram of the potential mechanism of EAPP2 inhibiting Syk in ACO inflammation. Data were shown as mean \pm SEM; ${ }^{\#} \mathrm{P}<0.01$ compared with the control group; ${ }^{*} \mathrm{P}<0.05$ and $* * \mathrm{P}<0.0 \mathrm{I}$ compared with the model group. 
and neutrophils infiltration in airways, important distinguishing markers of asthma and COPD, respectively. ${ }^{23}$ Furthermore, cytokines associated with Th1 and Th2 reactions were both increased, IFN- $\gamma$ associated with Th1, ${ }^{24}$ while IL-4 associated with Th2, which activated B lymphocytes to produce and secrete $\operatorname{IgE}$ and $\operatorname{IgG} 1{ }^{25}$ In this study, EAPP-2 significantly alleviated eosinophils, neutrophils and other inflammatory cells infiltration in lung tissues, as well as in BALF of ACO mice. EAPP-2 markedly reduced both Th1 (IFN- $\gamma$ ) and Th2 (IL-4) cytokine production in BALF, as well as inflammatory cytokines IL-6, IL-17A, TNF- $\alpha$ and IL-1 $\beta$, which being considered general markers of inflammation. ${ }^{26}$ Additionally, as a result of Th2 inhibition, EAPP-2 significantly inhibited the production of total IgE and IgG1 in serum. These results indicated that the enormous potential of EAPP-2 in ACO inflammation treatment.

The typical target of asthma and COPD inflammation, such as a target regulating both eosinophils and neutrophils, may be an effective target for ACO therapeutic intervention. Syk, a $72 \mathrm{kD}$ non-receptor protein tyrosine kinase, is widely present in various hematopoietic cells and some non-hematopoietic cells, including eosinophils, neutrophils, macrophages, epithelial cells, and so on. ${ }^{7-9}$ Syk is an essential regulator of immune signals, regulating multiple downstream signal transduction pathways, among which activated NF- $\mathrm{\kappa B}$ induces the expression of inflammatory genes iNOS, IL-1 $\beta$, IL-6, and TNF- $\alpha .{ }^{27}$ In asthma, Syk is rapidly recruited and activated by FceRI-mediated signaling pathways in human basophils, leading to cell degranulation and histamine release. ${ }^{12}$ Furthermore, in patients with COPD, Syk is one of the kinases required for neutrophils to form neutrophil extracellular traps (NET). ${ }^{13,14}$ Syk has been proved to be a potential therapeutic target for both asthma and COPD inflammation. ${ }^{10,11}$ So that, Syk seems to meet the potential target of ACO. This study showed that EAPP-2 treatment significantly inhibited the expression and phosphorylation of Syk in vivo and in vitro, and a synergistic effect with Syk inhibitor on IL-6 and TNF- $\alpha$ production was shown in vitro. These results indicate that Syk is a potential target that EAPP-2 regulated in ACO.

Furthermore, whether EAPP-2 is Syk targeted or targets its downstream was explored. Syk is involved in the activation of NLRP3 inflammasome, through controlling the interaction with caspase-1 or ASC. ${ }^{17,28-30}$ Activation of NLRP3 inflammasome usually requires both a priming signal and an activation signal, of which NF- $\mathrm{KB}$ acts as a central mediator of the priming signal by inducing the transcriptional expression of NLRP3 and pro-IL-1 $\beta^{31,32}$ In addition to activated NF$\kappa B$, mature IL-1 $\beta$ secretion depends on the NLRP3 inflammasomes. ${ }^{33}$ In this study, our results showed that EAPP-2 treatment also down-regulating the expression of NF- $\mathrm{B}, \mathrm{p}-\mathrm{NF}-\mathrm{\kappa B}$, and NLRP3 in vivo and in vitro. Moreover, the in vitro inhibition of inflammatory cytokines was synergistic with their inhibitors. Moreover, EAPP-2 treatment enhanced the inhibited effects on protein expression and phosphorylation with Syk, NF- $\mathrm{kB}$ and NLRP3 inhibitors. Other than that, Syk inhibitor enhanced the inhibition of EAPP-2 on NF- $\mathrm{KB}$ and NLRP3 expression and phosphorylation, while there were no enhanced effects of NF- $\mathrm{BB}$ and NLRP3 inhibitor on Syk expression and phosphorylation. This result indicates that EAPP-2 seems to target on Syk, thereby regulating NF- $\kappa \mathrm{B} / \mathrm{NLRP} 3$ signal transduction to inhibit the inflammatory response in ACO (Figure 6H).

In conclusion, EAPP-2 is shown to be a potentially useful therapeutic candidate for ACO, and its mechanism is at least partially achieved by targeting on Syk, and then inhibiting the NF- $\mathrm{kB}$ or NLRP3. Moreover, this study suggests that Syk may be a potentially effective target for ACO therapy.

\section{Data Sharing Statement}

The data that support the findings of this study are available from the corresponding author upon reasonable request.

\section{Ethics Approval and Consent to Participate}

Ethical approval of all animal experiments was obtained from the institutional guidelines at the Experimental Animal Center of the Institute of Materia Medica (IMM), Chinese Academy of Medical Sciences and Peking Union Medical College (CAMS and PUMC), approved by The Animal Care \&Welfare Committee of IMM, CAMS and PUMC, and conformed to internationally accepted ethical standards.

\section{Author Contributions}

All authors made a significant contribution to the work reported, whether that is in the conception, study design, execution, acquisition of data, analysis and interpretation, or in all these areas; took part in drafting, revising or 
critically reviewing the article; gave final approval of the version to be published; have agreed on the journal to which the article has been submitted; and agree to be accountable for all aspects of the work.

\section{Funding}

This work was financially supported by the National Natural Science Foundation of China (Grant No. 81973539), the Beijing Key Laboratory of New Drug Mechanisms and Pharmacological Evaluation Studies (BZ0150). The funders had no role in study design, data collection and analysis, decision to publish, or preparation of the manuscript.

\section{Disclosure}

The authors report no conflicts of interest in this work.

\section{References}

1. Global Initiative for Asthma - Global Initiative for Asthma - GINA. Available from: https://ginasthma.org/. Accessed March 26, 2020.

2. Global Initiative for Chronic Obstructive Lung Disease - Global Initiative for Chronic Obstructive Lung Disease - GOLD. Available from: https://goldcopd.org/. Accessed March 26, 2020.

3. Shantakumar S, Pwu R-F, D'Silva L, et al. Burden of asthma and COPD overlap (ACO) in Taiwan: a nationwide population-based study. BMC Pulm Med. 2018;18(1). doi:10.1186/s12890-017-0571-7

4. Roberto DM, Giancarlo P, Alessandro M, et al. The coexistence of asthma and chronic obstructive pulmonary disease (COPD): prevalence and risk factors in young, middle-aged and elderly people from the general population. PLoS One. 2013;8(5):e62985. doi:10.1371/ journal.pone.0062985

5. Chung W-S, Lin C-L, Kao C-H. Comparison of acute respiratory events between asthma-COPD overlap syndrome and COPD patients: a population-based cohort study. Medicine. 2015;94(17): e755. doi:10.1097/MD.0000000000000755

6. Kauppi P, Kupiainen H, Lindqvist A, et al. Overlap syndrome of asthma and COPD predicts low quality of life. J Asthma. 2011;48 (3):279-285. doi:10.3109/02770903.2011.555576

7. Tsuchida S, Yanagi S, Inatome $\mathrm{R}$, et al. Purification of a $72-\mathrm{kDa}$ protein-tyrosine kinase from rat liver and its identification as Syk: involvement of Syk in signaling events of hepatocytes. J Biochem. 2000;127(2):321-327. doi:10.1093/oxfordjournals.jbchem.a022610

8. Yamada T, Fujieda S, Yanagi S, et al. Protein-tyrosine kinase Syk expressed in human nasal fibroblasts and its effect on RANTES production. $J$ Immunol. 2001;166(1):538-543. doi:10.4049/ jimmunol.166.1.538

9. Fueyo J, Alonso MM, Parker Kerrigan BC, Gomez-Manzano C. Linking inflammation and cancer: the unexpected SYK world. Neuro Oncol. 2018;20(5):582-583. doi:10.1093/neuonc/noy036

10. Fan Y, Zhang Z, Yao C, et al. A derivative from resveratrol, ameliorates lipopolysaccharide/cigarette smoke-induced airway inflammation by blocking the Syk/NF- $\mathrm{BB}$ pathway. Front Pharmacol. 2019;10:1157. doi:10.3389/fphar.2019.01157

11. Yang H, Sun W, Ma P, et al. Multiple components rapidly screened from perilla leaves attenuate asthma airway inflammation by synergistic targeting on Syk. J Inflamm Res. 2020;13:897-911. doi:10.2147/JIR.S281393
12. Kepley CL, Youssef L, Andrews RP, Wilson BS, Oliver JM. Syk deficiency in nonreleaser basophils. $J$ Allergy Clin Immunol. 1999;104(2 Pt 1):279-284. doi:10.1016/s0091-6749(99)70367-2

13. Nanì S, Fumagalli L, Sinha U, Kamen L, Scapini P, Berton G. Src family kinases and Syk are required for neutrophil extracellular trap formation in response to $\beta$-glucan particles. J Innate Immun. 2015;7 (1):59-73. doi: $10.1159 / 000365249$

14. Elaskalani O, Abdol Razak NB, Metharom P. Neutrophil extracellular traps induce aggregation of washed human platelets independently of extracellular DNA and histones. Cell Commun Signal. 2018;16(1):24. doi:10.1186/s12964-018-0235-0

15. Ma P, Li S, Yang H, et al. Comparative RNA-Seq transcriptome analysis on pulmonary inflammation in a mouse model of asthmaCOPD overlap syndrome. Front Cell Dev Biol. 2021;9. doi:10.3389/ fcell.2021.628957

16. Liu D, Mamorska-Dyga A. Syk inhibitors in clinical development for hematological malignancies. J Hematol Oncol. 2017;10(1):145. doi:10.1186/s13045-017-0512-1

17. Lu Y-Q, Zhong S, Meng N, Fan Y-P, Tang W-X. NLRP3 inflammasome activation results in liver inflammation and fibrosis in mice infected with Schistosoma japonicum in a Syk-dependent manner. Sci Rep. 2017;7(1):8120. doi:10.1038/s41598-017-08689-1

18. Lu Y, Li X, Park YN, et al. Britanin suppresses IgE/Ag-induced mast cell activation by inhibiting the Syk pathway. Biomol Ther (Seoul). 2014;22(3):193-199. doi:10.4062/biomolther.2014.038

19. Bandgar BP, Patil SA, Korbad BL, Biradar SC, Nile SN, Khobragade CN. Synthesis and biological evaluation of a novel series of 2,2-bisaminomethylated aurone analogues as anti-inflammatory and antimicrobial agents. Eur J Med Chem. 2010;45(7):3223-3227. doi:10.1016/j.ejmech.2010.03.045

20. Hassan GS, Abou-Seri SM, Kamel G, Ali MM. Celecoxib analogs bearing benzofuran moiety as cyclooxygenase-2 inhibitors: design, synthesis and evaluation as potential anti-inflammatory agents. Eur $J$ Med Chem. 2014;76:482-493. doi:10.1016/j.ejmech.2014.02.033

21. Xie Y-S, Kumar D, Bodduri VDV, et al. Microwave-assisted parallel synthesis of benzofuran-2-carboxamide derivatives bearing anti-inflammatory, analgesic and antipyretic agents. Tetrahedron Lett. 2014;55(17):2796-2800. doi:10.1016/j.tetlet.2014.02.116

22. Yadav P, Singh P, Tewari AK. Design, synthesis, docking and anti-inflammatory evaluation of novel series of benzofuran based prodrugs. Bioorg Med Chem Lett. 2014;24(10):2251-2255. doi:10.1016/j.bmcl.2014.03.087

23. Iwamoto $\mathrm{H}$, Gao J, Koskela J, et al. Differences in plasma and sputum biomarkers between COPD and COPD-asthma overlap. Eur Respir J. 2014;43(2):421. doi:10.1183/09031936.00024313

24. Sethi S, Mahler DA, Marcus P, Owen CA, Yawn B, Rennard S. Inflammation in COPD: implications for management. Am J Med. 2012;125(12):1162-1170. doi:10.1016/j.amjmed.2012.06.024

25. Charrad R, Berraïs A, Hamdi B, Ammar J, Hamzaoui K, Hamzaoui A. Anti-inflammatory activity of IL-37 in asthmatic children: correlation with inflammatory cytokines TNF- $\alpha$, IL- $\beta$, IL-6 and IL-17A. Immunobiology. 2016;221(2):182-187. doi:10.1016/j.imbio.2015.09.009

26. Rincon M, Irvin CG. Role of IL-6 in asthma and other inflammatory pulmonary diseases. Int J Biol Sci. 2012;8(9):1281-1290. doi:10.7150/ijbs.4874

27. Hunto ST, Kim HG, Baek K-S, et al. Loratadine, an antihistamine drug, exhibits anti-inflammatory activity through suppression of the NF-kB pathway. Biochem Pharmacol. 2020;177:113949. doi:10.1016/j.bcp.2020.113949

28. Gross O, Poeck H, Bscheider M, et al. Syk kinase signalling couples to the Nlrp3 inflammasome for anti-fungal host defence. Nature. 2009;459(7245):433-436. doi:10.1038/nature07965

29. Saïd-Sadier N, Padilla E, Langsley G, Ojcius DM. Aspergillus fumigatus stimulates the NLRP3 inflammasome through a pathway requiring ROS production and the Syk tyrosine kinase. PLoS One. 2010;5 (4):e10008. doi:10.1371/journal.pone. 0010008 
30. Lin Y-C, Huang D-Y, Wang J-S, et al. Syk is involved in NLRP3 inflammasome-mediated caspase-1 activation through adaptor ASC phosphorylation and enhanced oligomerization. J Leukoc Biol. 2015;97(5):825-835. doi:10.1189/jlb.3HI0814-371RR

31. Qiao Y, Wang P, Qi J, Zhang L, Gao C. TLR-induced NF- $\kappa B$ activation regulates NLRP3 expression in murine macrophages. FEBS Lett. 2012;586(7):1022-1026. doi:10.1016/j.febslet.2012.02.045
32. Liu T, Zhang L, Joo D, Sun S-C. NF- $\mathrm{BB}$ signaling in inflammation. Signal Transduct Target Ther. 2017;2:17023. doi:10.1038/ sigtrans.2017.23

33. Latz E, Xiao TS, Stutz A. Activation and regulation of the inflammasomes. Nat Rev Immunol. 2013;13(6):397-411. doi: $10.1038 /$ nri3452

\section{Publish your work in this journal}

The Journal of Inflammation Research is an international, peerreviewed open-access journal that welcomes laboratory and clinical findings on the molecular basis, cell biology and pharmacology of inflammation including original research, reviews, symposium reports, hypothesis formation and commentaries on: acute/chronic inflammation; mediators of inflammation; cellular processes; molecular mechanisms; pharmacology and novel anti-inflammatory drugs; clinical conditions involving inflammation. The manuscript management system is completely online and includes a very quick and fair peerreview system. Visit http://www.dovepress.com/testimonials.php to read real quotes from published authors.

Submit your manuscript here: https://www.dovepress.com/journal-of-inflammation-research-journal 